\title{
A Fourth-Order Conservative Compact Finite Difference Scheme for the Generalized RLW Equation
}

\author{
Shuguang Li, Jue Wang, and Yuesheng Luo \\ School of Science, Harbin Engineering University, Harbin 150001, China \\ Correspondence should be addressed to Shuguang Li; 1sg9008@163.com and Jue Wang; wangjue3721@163.com
}

Received 31 October 2014; Accepted 29 January 2015

Academic Editor: Sandile Motsa

Copyright (c) 2015 Shuguang Li et al. This is an open access article distributed under the Creative Commons Attribution License, which permits unrestricted use, distribution, and reproduction in any medium, provided the original work is properly cited.

\begin{abstract}
The generalized regularized long-wave (GRLW) equation is studied by finite difference method. A new fourth-order energy conservative compact finite difference scheme was proposed. It is proved by the discrete energy method that the compact scheme is solvable, the convergence and stability of the difference schemes are obtained, and its numerical convergence order is $O\left(\tau^{2}+h^{4}\right)$ in the $L^{\infty}$-norm. Further, the compact schemes are conservative. Numerical experiment results show that the theory is accurate and the method is efficient and reliable.
\end{abstract}

\section{Introduction}

In this paper, we consider the following generalized regularized long-wave equation:

$$
u_{t}+u_{x}+\alpha\left(u^{p}\right)_{x}-\beta u_{x x t}=0, \quad(x, t) \in\left(x_{l}, x_{r}\right) \times(0, T]
$$

with the boundary conditions

$$
u\left(x_{l}, t\right)=u\left(x_{r}, t\right)=0, \quad t \in(0, T],
$$

and the initial condition

$$
u(x, 0)=u_{0}(x), \quad x \in\left(x_{l}, x_{r}\right),
$$

where $u=u(x, t)$ is a real-valued function defined on $\left(x_{l}, x_{r}\right) \times(0, T], \alpha, \beta>0, p \geq 2$ is a positive integer, and $u_{0}$ is a given function with Dirichlet boundary condition.

The GRLW equation was first put forward as a model for small-amplitude long waves on the surface of water in a channel by Peregrine $[1,2]$. A special case of (1), that is,

$$
u_{t}+u_{x}+\alpha u u_{x}-\beta u_{x x t}=0,
$$

is usually called the regularized long-wave (RLW) equation. The RLW equation is a representation form of nonlinear long wave and can describe a lot of important physical phenomena, such as shallow waves and ionic waves. The GRLW equation can also describe that wave motion to the same order of approximation as the KDV equation, so it plays a major role in the study of nonlinear dispersive waves [3]. It is difficult to find the analytical solution for (1), which has been studied by many researchers. The finite difference method for the initial-boundary value problem of the GRLW equation had been studied in [4-8]. Other mathematical theory and numerical methods for GRLW equation were considered in [9-11]. Reference [12] solved the GRLW equation by the Petrov-Galerkin method. Numerical solution of GRLW equation used Sinc-collocation method in [13]. In [14], a time-linearization method that uses a Crank-Nicolson procedure in time and three-point, fourth-order accurate in space, compact difference equations, is presented and used to determine the solutions of the generalized regularized-long wave (GRLW) equation. Recently, there has been growing interest in high-order compact methods for solving partial differential equations [15-17].

In this paper, we consider problem (1)-(3); it has the following conservation law:

$$
E(t)=\|u\|_{L^{2}}^{2}+\beta\left\|u_{x}\right\|_{L^{2}}^{2}=\text { const. }
$$

Using a customary designation, we will refer to the functional $E(t)$ as the energy integral, although it is not necessarily identifiable with energy in the original physical problem. 
We aim to present a conservative finite difference scheme for problem (1)-(3), which simulates conservation law (5) that the differential equation (1) possesses, and prove convergence and stability of the scheme. This paper is organized as follows. In Section 2, some notations are given and some useful lemmas are proposed. In Section 3, we present a nonlinear compact conservative difference scheme, discuss its discrete conservative law, prove the existence of difference solution by Brouwer fixed point theorem, give some a priori estimates, and then prove by discrete energy method that the difference scheme is uniquely solvable, unconditionally stable and that convergence of the difference solutions with $O\left(\tau^{2}+h^{4}\right)$ order is based on some a priori estimates. In Section 4 , numerical results are provided to test the theoretical results.

\section{Notations and Lemmas}

Let $h=\left(x_{r}-x_{l}\right) / J$ and $\tau=T / N$ be the spatial and temporal step sizes, respectively. Denote $x_{j}=x_{l}+j h,(0 \leq j \leq$ $J), t^{n}=n \tau,(0 \leq n \leq N)$. Let $u_{j}^{n}$ denote the approximation of $u\left(x_{j}, t_{n}\right)$, and let

$$
\mathbf{R}_{0}^{J}=\left\{u_{j}=\left(u_{j}\right)_{j \in \mathbb{Z}} \mid u_{0}=u_{J}=0\right\}
$$

As usual, the following notations will be used:

$$
\begin{gathered}
\delta_{x}^{+} u_{j}^{n}=\frac{u_{j+1}^{n}-u_{j}^{n}}{h}, \quad \delta_{x}^{-} u_{j}^{n}=\frac{u_{j}^{n}-u_{j-1}^{n}}{h}, \\
\delta_{x} u_{j}^{n}=\frac{u_{j+1}^{n}-u_{j-1}^{n}}{2 h}, \\
\bar{u}_{j}^{n}=\frac{u_{j}^{n+1}+u_{j}^{n-1}}{2}, \quad \delta_{t} u_{j}^{n}=\frac{u_{j}^{n+1}-u_{j}^{n-1}}{2 \tau}, \\
\mathscr{A}_{1} u_{j}^{n}=\left(1+\frac{h^{2}}{12} \delta_{x}^{+} \delta_{x}^{-}\right) u_{j}^{n}, \\
\mathscr{A}_{2} u_{j}^{n}=\left(1+\frac{h^{2}}{6} \delta_{x}^{+} \delta_{x}^{-}\right) u_{j}^{n} .
\end{gathered}
$$

We now introduce the discrete $L^{2}$-inner product and the associated norm

$$
\left(u^{n}, v^{n}\right)_{h}=h \sum_{j=1}^{J-1} u_{j}^{n} v_{j}^{n}, \quad\left\|u^{n}\right\|_{h}=\left(u^{n}, u^{n}\right)_{h}^{1 / 2} .
$$

The discrete $L^{\infty}$-norm $\|\cdot\|_{\infty, h}$ is defined as

$$
\|u\|_{\infty, h}=\max _{j}\left|u_{j}\right|
$$

Let $\Omega_{h}=\left\{x_{j}=x_{l}+j h \mid 0 \leq j \leq J\right\}$. It is convenient to let $L_{h}^{2}\left(\Omega_{h}\right)$ denote the normed vector space as $\left\{\mathbf{R}_{0}^{J},\|\cdot\|_{h}\right\}$. The corresponding matrices are defined, respectively, as

$$
\begin{gathered}
u^{n}=\left(u_{1}^{n}, u_{2}^{n}, \ldots, u_{J-1}^{n}\right)^{T}, \\
\left(u^{n}\right)^{p}=\operatorname{diag}\left(\left(u_{1}^{n}\right)^{p},\left(u_{2}^{n}\right)^{p}, \ldots,\left(u_{J-1}^{n}\right)^{p}\right), \\
A_{1}=\frac{1}{12}\left(\begin{array}{ccccc}
10 & 1 & 0 & \cdots & 0 \\
1 & 10 & 1 & \cdots & 0 \\
& \ddots & \ddots & \ddots & \\
0 & \cdots & 1 & 10 & 1 \\
0 & \cdots & 0 & 1 & 10
\end{array}\right)_{J-1}, \\
A_{2}=\frac{1}{6}\left(\begin{array}{ccccc}
4 & 1 & 0 & \cdots & 0 \\
1 & 4 & 1 & \cdots & 0 \\
& \ddots & \ddots & \ddots & \\
0 & \cdots & 1 & 4 & 1 \\
0 & \cdots & 0 & 1 & 4
\end{array}\right)_{J-1} .
\end{gathered}
$$
by

For a simple notation, the discrete function $\varphi$ is defined

$$
\varphi(u, v)=\frac{p \alpha}{p+1}\left[(u)^{p-1} H_{2} \delta_{x} v+H_{2} \delta_{x}\left(u^{p-1} v\right)\right],
$$

where $H_{1}=A_{1}^{-1}, H_{2}=A_{2}^{-1}$. Obviously, $A_{1}, A_{2}, H_{1}, H_{2}$ are symmetric positive definite matrices. To obtain some important results, we introduce the following lemmas.

Lemma 1 (see [18]). For $u, v \in \mathbf{R}_{0}^{J}$, one has

$$
\left(\delta_{x}^{+} u, v\right)_{h}=-\left(u, \delta_{x}^{-} v\right)_{h}, \quad\left(\delta_{x} u, v\right)_{h}=-\left(u, \delta_{x} v\right)_{h} .
$$

Lemma 2. For any real symmetric positive definite matrices $H$ and for $u, v \in \mathbf{R}_{0}^{J}$, one can get

$$
\begin{gathered}
\left(H \delta_{x}^{+} \delta_{x}^{-} u, v\right)_{h}=-\left(H \delta_{x}^{+} u, \delta_{x}^{+} v\right)_{h}=-\left(R \delta_{x}^{+} u, R \delta_{x}^{+} v\right)_{h}, \\
\left(H \delta_{x} u, v\right)_{h}=-\left(H u, \delta_{x} v\right)_{h}=-\left(u, H \delta_{x} v\right)_{h},
\end{gathered}
$$

where $R$ is obtained by Cholesky decomposition of $H$, denoted as $H=R^{T} R$.

Proof. For $u, v \in \mathbf{R}_{0}^{J}$, we have

$$
\begin{gathered}
\left(H \delta_{x}^{+} \delta_{x}^{-} u, v\right)_{h}=\left(\delta_{x}^{+} \delta_{x}^{-} H u, v\right)_{h}=-\left(\delta_{x}^{+} H u, \delta_{x}^{+} v\right)_{h} \\
=-\left(H \delta_{x}^{+} u, \delta_{x}^{+} v\right)_{h}=-\left(R^{T} R \delta_{x}^{+} u, \delta_{x}^{+} v\right)_{h} \\
=-\left(R \delta_{x}^{+} u, R \delta_{x}^{+} v\right)_{h}, \\
\left(H \delta_{x} u, v\right)_{h}=\left(\delta_{x} H u, v\right)_{h}=-\left(H u, \delta_{x} v\right)_{h}=-\left(u, H \delta_{x} v\right)_{h} .
\end{gathered}
$$


Lemma 3 (see [16]). On the matrices $A_{1}, A_{2}$. The eigenvalues of the matrices $A_{1}$ and $A_{2}$ are, respectively, as follows:

$$
\begin{aligned}
& \lambda_{A_{1}, i}=\frac{1}{6}\left(5+\cos \frac{2 i \pi}{J}\right), \quad \lambda_{A_{2}, i}=\frac{1}{3}\left(2+\cos \frac{2 i \pi}{J}\right), \\
& i=1,2, \ldots, J-1 \text {. }
\end{aligned}
$$

Lemma 4. For $u \in \mathbf{R}_{0}^{J}$, we can get

$$
\begin{gathered}
\|u\|_{h}^{2} \leq\left(H_{1} u, u\right)_{h}=\left\|R_{1} u\right\|_{h}^{2} \leq \frac{3}{2}\|u\|_{h}^{2}, \\
\|u\|_{h}^{2} \leq\left(H_{2} u, u\right)_{h}=\left\|R_{2} u\right\|_{h}^{2} \leq 3\|u\|_{h}^{2},
\end{gathered}
$$

where $R_{l}$ are obtained by Cholesky decomposition of $H_{l}$, denoted as $H_{l}=R_{l}^{T} R_{l}, \quad(l=1,2)$.

Proof. It follows from Lemma 3 that the eigenvalues of $H_{1}$ and $\mathrm{H}_{2}$ satisfy

$$
\begin{aligned}
& 1 \leq \lambda_{H_{1}, k} \leq \frac{3}{2}, \quad k=1,2, \ldots, J-1, \\
& 1 \leq \lambda_{H_{2}, k} \leq 3, \quad k=1,2, \ldots, J-1 .
\end{aligned}
$$

This gives the spectral radius $\rho\left(H_{1}\right) \leq 3 / 2, \rho\left(H_{2}\right) \leq 3$, and consequently

$$
1 \leq\left\|H_{1}\right\|=\rho\left(H_{1}\right) \leq \frac{3}{2}, \quad 1 \leq\left\|H_{2}\right\|=\rho\left(H_{2}\right) \leq 3 .
$$

Thus

$$
\begin{gathered}
\|u\|_{h}^{2} \leq\left(H_{1} u, u\right)_{h}=\left(R_{1} u, R_{1} u\right)_{h} \leq\left\|H_{1}\right\|(u, u)_{h} \leq \frac{3}{2}\|u\|_{h}^{2}, \\
\|u\|_{h}^{2} \leq\left(H_{2} u, u\right)_{h}=\left(R_{2} u, R_{2} u\right)_{h} \leq\left\|H_{2}\right\|(u, u)_{h} \leq 3\|u\|_{h}^{2} .
\end{gathered}
$$

Lemma 5. For $u, v \in \mathbf{R}_{0}^{J}$, one has

$$
(\varphi(u, v), v)_{h}=0 \text {. }
$$

Proof. For $u \in \mathbf{R}_{0}^{J}$, we have

$$
\begin{aligned}
& (\varphi(u, v), v)_{h} \\
& \quad=\frac{p \alpha}{p+1}\left(u^{p-1} H_{2} \delta_{x} v+H_{2} \delta_{x}\left(u^{p-1} v\right), v\right)_{h} \\
& =\frac{p \alpha}{p+1}\left[\left(u^{p-1} H_{2} \delta_{x} v, v\right)_{h}+\left(H_{2} \delta_{x}\left(u^{p-1} v\right), v\right)_{h}\right] \\
& =\frac{p \alpha}{p+1}\left[\left(H_{2} \delta_{x} v, u^{p-1} v\right)_{h}-\left(u^{p-1} v, H_{2} \delta_{x} v\right)_{h}\right]=0 .
\end{aligned}
$$

Lemma 6 (see [18]). For any discrete function $u \in \mathbf{R}_{0}^{J}$ and for any given $\varepsilon>0$, there exists a constant $K(\varepsilon, n)$, depending only on $\varepsilon$ and $n$, such that

$$
\left\|u^{n}\right\|_{\infty, h} \leq \varepsilon\left\|\delta_{x}^{+} u^{n}\right\|_{h}+K(\varepsilon, n)\left\|u^{n}\right\|_{h} .
$$

Lemma 7 (see [19]). Let $(H,(\cdot, \cdot))$ be a finite-dimensional inner product space, let $\|\cdot\|$ be the associated norm, and let $g: H \rightarrow H$ be continuous. Assume, moreover, that $\exists \alpha>0$, $\forall z \in H,\|z\|=\alpha,(g(z), z)>0$. Then, there exists a $z^{*} \in H$ such that $g\left(z^{*}\right)=0$ and $\left\|z^{*}\right\| \leq \alpha$.

Lemma 8 (see [18]). Suppose that the discrete function $w_{h}$ satisfies recurrence formula

$$
w_{n}-w_{n-1} \leq A \tau w_{n}+B \tau w_{n-1}+C_{n} \tau,
$$

where $A, B$, and $C_{n}(n=1, \ldots, N)$ are nonnegative constants. Then

$$
\left\|w_{h}\right\| \leq\left(w_{0}+\tau \sum_{k=1}^{N} C_{k}\right) e^{2(A+B) \tau},
$$

where $\tau$ is sufficiently small, such that $(A+B) \tau \leq(N-$ $1) / 2 N(N>1)$.

\section{A Nonlinear-Implicit Conservative Scheme}

In this section, we propose a nonlinear-implicit conservative scheme for the initial-boundary value problem (1)-(3) and give its numerical analysis.

3.1. The Nonlinear-Implicit Scheme and Its Conservative Law. Next we consider the compact finite difference scheme for problem (1)-(3) as follows:

$$
\begin{gathered}
\partial_{t} u_{j}^{n}+\theta \mathscr{A}_{2}^{-1} \delta_{x} u_{j}^{n}+(1-\theta) \mathscr{A}_{2}^{-1} \delta_{x} \bar{u}_{j}^{n} \\
+\frac{p \alpha}{p+1}\left[\left(\bar{u}_{j}^{n}\right)^{p-1} \mathscr{A}_{2}^{-1} \delta_{x} \bar{u}_{j}^{n}+\mathscr{A}_{2}^{-1} \delta_{x}\left(\bar{u}_{j}^{n}\right)^{p}\right] \\
-\beta \mathscr{A}_{1}^{-1} \delta_{x}^{+} \delta_{x}^{-}\left(\partial_{t} u_{j}^{n}\right)=0, \quad 1 \leq j \leq J-1, \quad 1 \leq n \leq N-1, \\
u_{0}^{n}=u_{J}^{n}=0, \quad 1 \leq n \leq N, \\
u_{j}^{0}=u_{0}\left(x_{j}\right), \quad 0 \leq j \leq J,
\end{gathered}
$$

where weight coefficient $\theta \in[0,1]$. Note that we need another finite difference scheme to calculate $u^{1}$, so the following scheme will be used:

$$
\begin{aligned}
u_{j}^{1}-\beta \mathscr{A}_{1}^{-1} \delta_{x}^{+} \delta_{x}^{-} u_{j}^{1}= & u_{0}\left(x_{j}\right)-\beta \frac{\partial^{2} u_{0}}{\partial x^{2}}\left(x_{j}\right)-\tau \frac{\partial u_{0}}{\partial x}\left(x_{j}\right) \\
& -\tau u_{0}\left(x_{j}\right) \frac{\partial u_{0}}{\partial x}\left(x_{j}\right) .
\end{aligned}
$$

The matrix form of the difference scheme (25) can be written as

$$
\begin{aligned}
\partial_{t} u^{n} & +\theta H_{2} \delta_{x} u^{n}+(1-\theta) H_{2} \delta_{x} \bar{u}^{n} \\
+ & \frac{p \alpha}{p+1}\left[\left(\bar{u}^{n}\right)^{p-1} H_{2} \delta_{x} \bar{u}^{n}+H_{2} \delta_{x}\left(\bar{u}^{n}\right)^{p}\right] \\
& -\beta H_{1} \delta_{x}^{+} \delta_{x}^{-}\left(\partial_{t} u^{n}\right)=0, \quad 1 \leq n \leq N-1,
\end{aligned}
$$




$$
\begin{array}{ll}
u_{0}^{n}=u_{J}^{n}=0, & 1 \leq n \leq N, \\
u_{j}^{0}=u_{0}\left(x_{j}\right), & 0 \leq j \leq J .
\end{array}
$$

Theorem 9. Suppose that $u_{0} \in H_{0}^{1}(\Omega)$; then the finite difference scheme (25) is conservative for discrete energy; that is,

$$
\begin{aligned}
E^{n}= & \frac{1}{2}\left(\left\|u^{n+1}\right\|_{h}^{2}+\left\|u^{n}\right\|_{h}^{2}\right)+\frac{\beta}{2}\left(\left\|R_{1} \delta_{x}^{+} u^{n+1}\right\|_{h}^{2}+\left\|R_{1} \delta_{x}^{+} u^{n}\right\|_{h}^{2}\right) \\
& +\tau \theta\left(R_{2} \delta_{x} u^{n}, R_{2} u^{n+1}\right)=E^{n-1}=\cdots=E^{0},
\end{aligned}
$$

where $R_{l}$ are obtained by Cholesky decomposition of $H_{l}$, denoted as $H_{l}=R_{l}^{T} R_{l}, \quad(l=1,2)$.

Proof. Taking an inner product of (27) with $u^{n+1}+u^{n-1}$, from Lemma 5, we obtain

$$
\begin{gathered}
\frac{1}{2 \tau}\left(\left\|u^{n+1}\right\|_{h}^{2}-\left\|u^{n-1}\right\|_{h}^{2}\right)+\theta\left(H_{2} \delta_{x} u^{n}, 2 \bar{u}^{n}\right)_{h} \\
+\frac{\beta}{2 \tau}\left(\left\|R_{1} \delta_{x}^{+} u^{n+1}\right\|_{h}^{2}-\left\|R_{1} \delta_{x}^{+} u^{n-1}\right\|_{h}^{2}\right)=0 .
\end{gathered}
$$

Noting that

$$
\begin{aligned}
\left(H_{2} \delta_{x} u^{n}, u^{n+1}+u^{n-1}\right)_{h}= & \left(R_{2} \delta_{x} u^{n}, R_{2} u^{n+1}\right)_{h} \\
& -\left(R_{2} \delta_{x} u^{n-1}, R_{2} u^{n}\right)_{h},
\end{aligned}
$$

from (31)-(32), we obtain

$$
\begin{aligned}
& \frac{1}{2 \tau}\left(\left\|u^{n+1}\right\|_{h}^{2}-\left\|u^{n-1}\right\|_{h}^{2}\right) \\
& +\theta\left[\left(R_{2} \delta_{x} u^{n}, R_{2} u^{n+1}\right)_{h}-\left(R_{2} \delta_{x} u^{n-1}, R_{2} u^{n}\right)_{h}\right] \\
& +\frac{\beta}{2 \tau}\left(\left\|R_{1} \delta_{x}^{+} u^{n+1}\right\|_{h}^{2}-\left\|R_{1} \delta_{x}^{+} u^{n-1}\right\|_{h}^{2}\right)=0 .
\end{aligned}
$$

Let

$$
\begin{aligned}
E^{n}= & \frac{1}{2}\left(\left\|u^{n+1}\right\|_{h}^{2}+\left\|u^{n}\right\|_{h}^{2}\right) \\
& +\frac{\beta}{2}\left(\left\|R_{1} \delta_{x}^{+} u^{n+1}\right\|_{h}^{2}+\left\|R_{1} \delta_{x}^{+} u^{n}\right\|_{h}^{2}\right) \\
& +\tau \theta\left(R_{2} \delta_{x} u^{n}, R_{2} u^{n+1}\right) .
\end{aligned}
$$

Then, from (33), we get $E^{n}=E^{n-1}$. This completes the proof.

\subsection{Existence and Prior Estimates of Difference Solution}

Theorem 10. Suppose that $u_{0} \in H_{0}^{1}(\Omega)$; then the finite difference scheme (25) is solvable.
Proof. Assume that there exist $u^{0}, u^{1}, \ldots, u^{n}$ which satisfy (25) as $n \leq N-1$; now we try to prove that $u^{n+1}$ satisfy (25). We define the mapping $g: \mathbf{R}_{0}^{J} \rightarrow \mathbf{R}_{0}^{J}$ as follows:

$$
\begin{aligned}
g(v)= & v-u^{n}-\beta\left(H_{1} \delta_{x}^{+} \delta_{x}^{-} v-H_{1} \delta_{x}^{+} \delta_{x}^{-} u^{n}\right) \\
& +\tau H_{2} \delta_{x} v+\tau \varphi(v, v)
\end{aligned}
$$

is obviously continuous. Taking in (35) the inner product with $v$, from Lemmas 2, 4, and 5, we obtain

$$
\begin{aligned}
& (g(v), v)_{h} \\
& \quad=\|v\|_{h}^{2}-\left(v, u^{n}\right)_{h}+\beta\left[\left\|R_{1} \delta_{x}^{+} v\right\|_{h}^{2}-\left(R_{1} \delta_{x}^{+} v, R_{1} \delta_{x}^{+} u^{n}\right)_{h}\right] \\
& \quad \geq \frac{1}{2}\left(\|v\|_{h}^{2}-\left\|u^{n}\right\|_{h}^{2}\right)+\frac{\beta}{2}\left(\left\|R_{1} \delta_{x}^{+} v\right\|_{h}^{2}-\left\|R_{1} \delta_{x}^{+} u^{n}\right\|_{h}^{2}\right) \\
& \quad \geq \frac{1}{2}\left(\|v\|_{h}^{2}-\left\|u^{n}\right\|_{h}^{2}\right)-\frac{3 \beta}{4}\left\|\delta_{x}^{+} u^{n}\right\|_{h}^{2} .
\end{aligned}
$$

Thus for $\|v\|_{h}^{2}=\left\|u^{n}\right\|_{h}^{2}+(3 \beta / 2)\left\|\delta_{x}^{+} u^{n}\right\|_{h}^{2}+1$, there exists $(g(v), v)_{h}>0$. The existence of $\bar{u}^{n}$ follows from Lemma 7 and consequently the existence of $u^{n+1}=2 v-u^{n-1}$ is obtained. This completes the proof.

Next we will give some a priori estimates of difference solutions.

Lemma 11. Suppose that $u_{0} \in H_{0}^{1}(\Omega)$; then there exists the estimation for the solution of problem (1)-(3):

$$
\|u\|_{L^{2}} \leq C, \quad\left\|u_{x}\right\|_{L^{2}} \leq C, \quad\|u\|_{L^{\infty}} \leq K_{0} .
$$

Proof. It follows from (5) that

$$
\|u\|_{L^{2}} \leq C, \quad\left\|u_{x}\right\|_{L^{2}} \leq C .
$$

Hence, it follows from the Sobolev inequality that

$$
\|u\|_{L^{\infty}} \leq K_{0} .
$$

Lemma 12. Suppose that $u_{0} \in H_{0}^{1}(\Omega)$; then there exists the estimation for the solution of the difference scheme (25):

$$
\left\|u^{n}\right\|_{h} \leq K_{1}, \quad\left\|\delta_{x}^{+} u^{n}\right\|_{h} \leq K_{2}, \quad\left\|u^{n}\right\|_{\infty, h} \leq K_{3},
$$

where $K_{4}=\max \left\{\sqrt{6 E^{0} /(3-5 \theta \tau)}, \sqrt{2 E^{0}}\right\}, K_{2}=$ $\max \left\{\sqrt{6 E^{0} /(3 \beta-5 \theta \tau)}, \sqrt{2 E^{0} / \beta}\right\}, K_{3}=\varepsilon K_{2}+K(\varepsilon, n) K_{1}$.

Proof. From Theorem 9, we obtain

$$
\begin{aligned}
& \frac{1}{2}\left(\left\|u^{n+1}\right\|_{h}^{2}+\left\|u^{n}\right\|_{h}^{2}\right)+\frac{\beta}{2}\left(\left\|R_{1} \delta_{x}^{+} u^{n+1}\right\|_{h}^{2}+\left\|R_{1} \delta_{x}^{+} u^{n}\right\|_{h}^{2}\right) \\
& \leq E^{0}+\tau \theta\left|\left(R_{2} \delta_{x} u^{n}, R_{2} u^{n+1}\right)_{h}\right| \\
& \leq E^{0}+\frac{\tau \theta}{2}\left(\left\|R_{2} \delta_{x} u^{n}\right\|_{h}^{2}+\left\|R_{2} u^{n+1}\right\|_{h}^{2}\right) ;
\end{aligned}
$$


then from Lemma 4, we have

$$
\begin{aligned}
& \frac{1}{2}\left(\left\|u^{n+1}\right\|_{h}^{2}+\left\|u^{n}\right\|_{h}^{2}\right)+\frac{\beta}{2}\left(\left\|\delta_{x}^{+} u^{n+1}\right\|_{h}^{2}+\left\|\delta_{x}^{+} u^{n}\right\|_{h}^{2}\right) \\
& \leq E^{0}+\frac{3 \tau \theta}{2}\left(\left\|\delta_{x}^{+} u^{n}\right\|_{h}^{2}+\left\|u^{n+1}\right\|_{h}^{2}\right) .
\end{aligned}
$$

That is

$$
\begin{aligned}
& \left(\frac{1}{2}-\frac{3 \tau \theta}{2}\right)\left\|u^{n+1}\right\|_{h}^{2}+\frac{1}{2}\left\|u^{n}\right\|_{h}^{2}+\frac{\beta}{2}\left\|\delta_{x}^{+} u^{n+1}\right\|^{2} \\
& +\left(\frac{\beta}{2}-\frac{3 \tau \theta}{2}\right)\left\|\delta_{x}^{+} u^{n}\right\|^{2} \leq E^{0}
\end{aligned}
$$

let $\tau$ be small, such that $\min \{1 / 2-3 \tau \theta / 2, \beta / 2-3 \tau \theta / 2\}>0$; then we can get

$$
\left\|u^{n}\right\|_{h} \leq K_{1}, \quad\left\|\delta_{x}^{+} u^{n}\right\|_{h} \leq K_{2},
$$

where

$$
\begin{aligned}
& K_{1}=\max \left\{\sqrt{\frac{2 E^{0}}{1-3 \theta \tau}}, \sqrt{2 E^{0}}\right\}, \\
& K_{2}=\max \left\{\sqrt{\frac{2 E^{0}}{\beta-3 \theta \tau}}, \sqrt{\frac{2 E^{0}}{\beta}}\right\} .
\end{aligned}
$$

It follows from Lemma 6 that

$$
\left\|u^{n}\right\|_{\infty, h} \leq K_{3},
$$

where $K_{3}=\varepsilon K_{2}+K(\varepsilon, n) K_{1}$.

This completes the proof.

3.3. Convergence and Stability of Difference Solution. First, we consider the truncation error of the finite difference scheme (25). Suppose that $v_{j}^{n}=u\left(x_{j}, t_{n}\right)$, which is the solution of problem (1)-(3). Then we have

$$
\begin{aligned}
r^{n}= & \partial_{t} v^{n}+\theta H_{2} \delta_{x} v^{n}+(1-\theta) H_{2} \delta_{x} \bar{v}^{n}-\beta H_{1} \delta_{x}^{+} \delta_{x}^{-} \partial_{t} v^{n} \\
& +\frac{p \alpha}{p+1}\left[\left(\bar{v}^{n}\right)^{p-1} H_{2} \delta_{x} \bar{v}^{n}+H_{2} \delta_{x}\left(\bar{v}^{n}\right)^{p}\right]
\end{aligned}
$$

according to Taylor's expansion, $r_{j}^{n}=O\left(\tau^{2}+h^{4}\right)$ can be easily obtained. Next, we consider convergence and stability of the finite difference scheme (25).

Theorem 13. Suppose that $u_{0} \in H_{0}^{1}(\Omega)$ and $u \in C^{(5,3)}$; then the solution of the conservative difference scheme (25) converges to the solution of problem (1)-(3) with the order $O\left(\tau^{2}+h^{4}\right)$ by $L^{\infty}$ norm.

Proof. Subtracting (27) from (47), and letting $e^{n}=v^{n}-u^{n}$, we have

$$
\begin{aligned}
r^{n}= & \partial_{t} e^{n}+\theta H_{2} \delta_{x} e^{n}+(1-\theta) H_{2} \delta_{x} \bar{e}^{n} \\
& -\beta H_{1} \delta_{x}^{+} \delta_{x}^{-} \partial_{t} e^{n}+\varphi\left(\bar{v}^{n}, \bar{v}^{n}\right)-\varphi\left(\bar{u}^{n}, \bar{u}^{n}\right) ;
\end{aligned}
$$

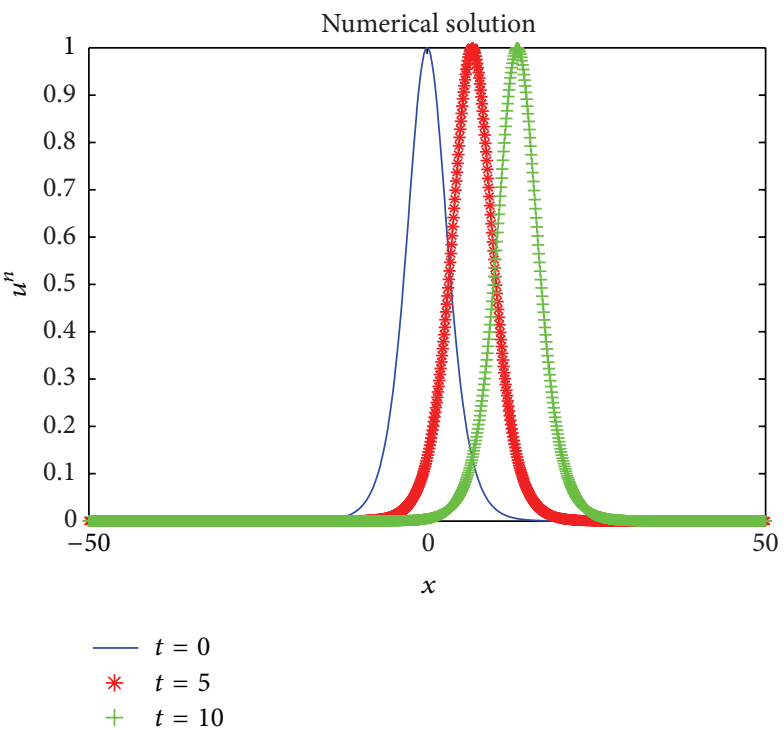

FIGURE 1: Numerical solution $u^{n}$ of scheme with $p=2$ and $\tau=h=$ 0.1 .

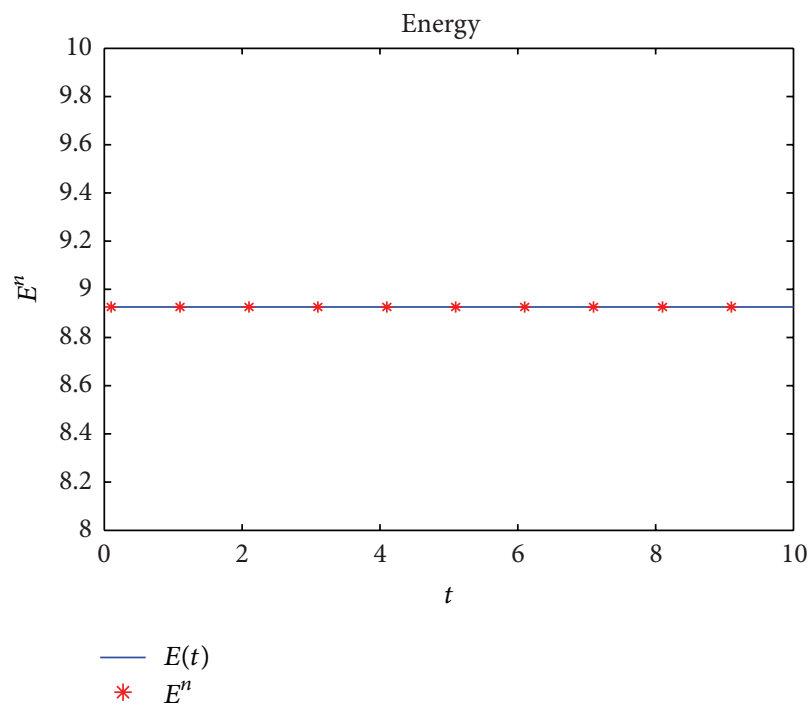

FIgURE 2: Discrete energy $E^{n}$ of scheme with $p=2, T=10$, and $\tau=h=0.1$.

taking an inner product of (48) with $e^{n+1}+e^{n-1}$, we obtain

$$
\begin{aligned}
\left(r^{n}, e^{n+1}+e^{n-1}\right)_{h} & \\
= & \frac{1}{2 \tau}\left(\left\|e^{n+1}\right\|_{h}^{2}-\left\|e^{n-1}\right\|_{h}^{2}\right) \\
& +\frac{\beta}{2 \tau}\left(\left\|R_{1} \delta_{x}^{+} e^{n+1}\right\|_{h}^{2}-\left\|R_{1} \delta_{x}^{+} e_{x}^{n-1}\right\|_{h}^{2}\right) \\
& +\theta\left(R_{2} \delta_{x} e^{n}, e^{n+1}+e^{n-1}\right)_{h} \\
& +\left(\varphi\left(\bar{v}^{n}, \bar{v}^{n}\right)-\varphi\left(\bar{u}^{n}, \bar{u}^{n}\right), e^{n+1}+e^{n-1}\right)_{h} ;
\end{aligned}
$$


TABLE 1: Errors computed by the proposed compact scheme with $\sigma=4 / 3, p=2$ and $h=\tau=0.1$.

\begin{tabular}{lccccc}
\hline$t$ & & & $\left\|e^{n}\right\|_{\infty, h}$ & $\theta=0.75$ & $\theta=1$ \\
\hline 0.2 & $\theta=0$ & $\theta=0.25$ & $\theta=0.5$ & $3.2838 \times 10^{-5}$ & $5.0401 \times 10^{-5}$ \\
0.4 & $5.6213 \times 10^{-5}$ & $1.2528 \times 10^{-5}$ & $1.5794 \times 10^{-5}$ & $6.5530 \times 10^{-5}$ & $1.0012 \times 10^{-4}$ \\
0.6 & $7.8509 \times 10^{-5}$ & $2.4917 \times 10^{-5}$ & $3.1520 \times 10^{-5}$ & $9.7948 \times 10^{-5}$ & $1.4917 \times 10^{-4}$ \\
0.8 & $1.0476 \times 10^{-4}$ & $3.7267 \times 10^{-5}$ & $4.7095 \times 10^{-5}$ & $1.3006 \times 10^{-4}$ & $1.9758 \times 10^{-4}$ \\
1.0 & $1.3090 \times 10^{-4}$ & $4.9503 \times 10^{-5}$ & $6.2474 \times 10^{-5}$ & $1.6222 \times 10^{-4}$ & $2.4638 \times 10^{-4}$ \\
\hline
\end{tabular}

TABLE 2: Errors computed by the proposed compact scheme with $\sigma=4 / 3, p=4$ and $h=\tau=0.1$.

\begin{tabular}{|c|c|c|c|c|c|}
\hline \multirow{2}{*}{$t$} & \multicolumn{5}{|c|}{$\left\|e^{n}\right\|_{\infty, h}$} \\
\hline & $\theta=0$ & $\theta=0.25$ & $\theta=0.5$ & $\theta=0.75$ & $\theta=1$ \\
\hline 0.2 & $1.2498 \times 10^{-4}$ & $1.7684 \times 10^{-4}$ & $2.3183 \times 10^{-4}$ & $2.8729 \times 10^{-4}$ & $3.4464 \times 10^{-4}$ \\
\hline 0.4 & $2.3629 \times 10^{-4}$ & $3.4550 \times 10^{-4}$ & $4.5445 \times 10^{-4}$ & $5.6499 \times 10^{-4}$ & $6.7531 \times 10^{-4}$ \\
\hline 0.6 & $3.3108 \times 10^{-4}$ & $5.0029 \times 10^{-4}$ & $6.6455 \times 10^{-4}$ & $8.3064 \times 10^{-4}$ & $9.9436 \times 10^{-4}$ \\
\hline 0.8 & $4.1205 \times 10^{-4}$ & $6.4180 \times 10^{-4}$ & $8.6651 \times 10^{-4}$ & $1.0913 \times 10^{-3}$ & $1.3159 \times 10^{-3}$ \\
\hline 1.0 & $4.9896 \times 10^{-4}$ & $7.7376 \times 10^{-4}$ & $1.0667 \times 10^{-3}$ & $1.3595 \times 10^{-3}$ & $1.6550 \times 10^{-3}$ \\
\hline
\end{tabular}

from Lemma 4 and Cauchy-Schwarz inequality, we obtain

$$
\begin{aligned}
& \left(R_{2} \delta_{x} e^{n}, e^{n+1}+e^{n-1}\right)_{h} \\
& \quad \leq 3\left\|\delta_{x} e_{x}^{n}\right\|_{h}^{2}+\frac{1}{2}\left\|e^{n+1}\right\|_{h}^{2}+\frac{1}{2}\left\|e^{n-1}\right\|_{h}^{2}
\end{aligned}
$$

according to Lemmas 2 and 4, we have

$$
\begin{aligned}
& \left(\varphi\left(\bar{v}^{n}, \bar{v}^{n}\right)-\varphi\left(\bar{u}^{n}, \bar{u}^{n}\right), e^{n+1}+e^{n-1}\right)_{h} \\
& =\frac{2 p \alpha}{p+1}\left\{h \sum_{j=1}^{J-1}\left[\left(\bar{v}_{j}^{n}\right)^{p-1} \mathscr{A}_{2}^{-1} \delta_{x} \bar{v}_{j}^{n}-\left(\bar{u}_{j}^{n}\right)^{p-1} \mathscr{A}_{2}^{-1} \delta_{x} \bar{u}_{j}^{n}\right] \bar{e}_{j}^{n}\right. \\
& \left.+h \sum_{j=0}^{J-1}\left[\mathscr{A}_{2}^{-1} \delta_{x}\left(\bar{v}_{j}^{n}\right)^{p}-\mathscr{A}_{2}^{-1} \delta_{x}\left(\bar{u}_{j}^{n}\right)^{p}\right] \bar{e}_{j}^{n}\right\} \\
& \leq K_{4} h \sum_{j=0}^{J-1}\left(\left|\mathscr{A}_{2}^{-1} \delta_{x} \bar{e}_{j}^{n}\right|+\left|\bar{e}_{j}^{n}\right|\right)\left|\bar{e}_{j}^{n}\right| \\
& +K_{4} h \sum_{j=0}^{J-1}\left|\bar{e}_{j}^{n}\right|\left|\mathscr{A}_{2}^{-1} \delta_{x} \bar{e}_{j}^{n}\right| \\
& \leq K_{4}\left(9\left\|\delta_{x} \bar{e}^{n}\right\|_{h}^{2}+2\left\|e^{n}\right\|_{h}^{2}\right) \\
& \leq K_{4}\left(\frac{9}{2}\left\|\delta_{x} e^{n+1}\right\|_{h}^{2}+\frac{9}{2}\left\|\delta_{x} e^{n-1}\right\|_{h}^{2}+\left\|e^{n+1}\right\|_{h}^{2}+\left\|e^{n-1}\right\|_{h}^{2}\right)
\end{aligned}
$$

where $K_{4}=(p \alpha / 2(p+1)) \max \left\{K_{0}^{p-1}, 2(p-1) K_{0}^{p-2} K_{3}, 2(p-\right.$ 1) $\left.K_{3}^{p-1}\right\}$.

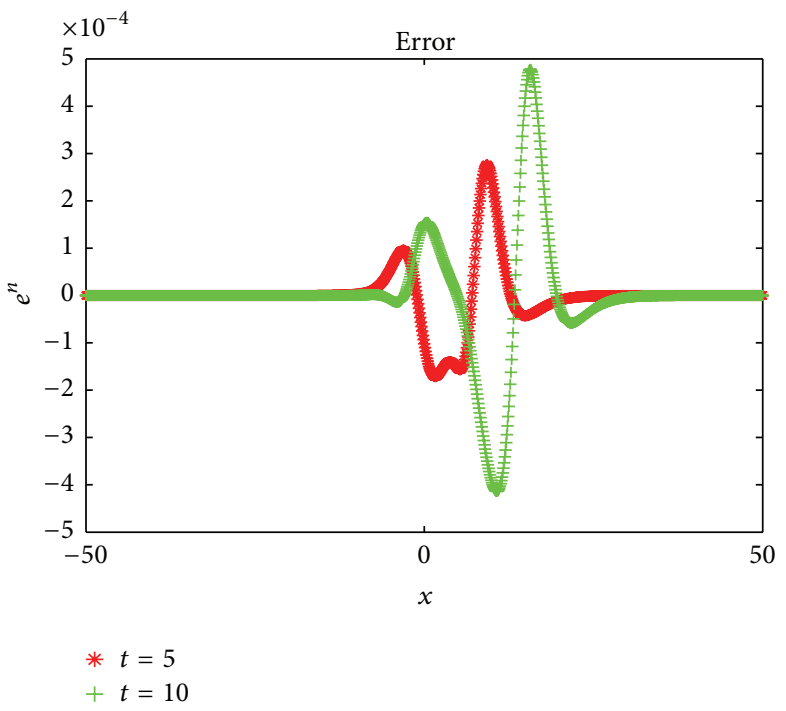

FIGURE 3: Absolute error $e^{N}$ of scheme with $p=2$ and $\tau=h=0.1$.

Substituting (50) and (51) into (49), we obtain

$$
\begin{aligned}
\frac{1}{2 \tau}\left(\left\|e^{n+1}\right\|_{h}^{2}-\left\|e^{n-1}\right\|_{h}^{2}\right)+\frac{\beta}{2 \tau}\left(\left\|R_{1} \delta_{x}^{+} e^{n+1}\right\|_{h}^{2}-\left\|R_{1} \delta_{x}^{+} e_{x}^{n-1}\right\|_{h}^{2}\right) \\
\leq\left\|r^{n}\right\|_{h}^{2}+\frac{1}{2}\left(\left\|e^{n+1}\right\|_{h}^{2}+\left\|e^{n-1}\right\|_{h}^{2}\right) \\
+\theta\left(3\left\|\delta_{x} e_{x}^{n}\right\|_{h}^{2}+\frac{1}{2}\left\|e^{n+1}\right\|_{h}^{2}+\frac{1}{2}\left\|e^{n-1}\right\|_{h}^{2}\right) \\
+K_{4}\left(\frac{9}{2}\left\|\delta_{x} e^{n+1}\right\|_{h}^{2}+\frac{9}{2}\left\|\delta_{x} e^{n-1}\right\|_{h}^{2}+\left\|e^{n+1}\right\|_{h}^{2}+\left\|e^{n-1}\right\|_{h}^{2}\right),
\end{aligned}
$$


TABLE 3: Comparison of $\|e\|_{\infty, h}$ by the compact scheme for $h=\tau=0.1$ with the Zhang [4] scheme for $h=0.05, \tau=0.1$.

\begin{tabular}{lcccc}
\hline$t$ & & & & $p=4$ \\
& Compact Scheme & Zhang [4] & Compact Scheme & Zhang [4] \\
\hline 0.2 & $1.2528 \times 10^{-5}$ & $7.756 \times 10^{-6}$ & $1.2498 \times 10^{-4}$ & 0.0049 \\
0.4 & $2.4917 \times 10^{-5}$ & $1.575 \times 10^{-5}$ & $2.3629 \times 10^{-4}$ & 0.0098 \\
0.6 & $3.7267 \times 10^{-5}$ & $2.357 \times 10^{-5}$ & $3.3108 \times 10^{-4}$ & 0.0148 \\
0.8 & $4.9503 \times 10^{-5}$ & $3.129 \times 10^{-5}$ & $4.1205 \times 10^{-4}$ & 0.0198 \\
1.0 & $6.1653 \times 10^{-5}$ & $3.963 \times 10^{-5}$ & $4.9896 \times 10^{-4}$ & 0.0239 \\
\hline
\end{tabular}

TABLE 4: Comparison of $\|e\|_{\infty, h}$ by the compact scheme for $\sigma=4 / 3, \theta=0.25, p=2, \tau=0.1$ and $h=0.05$.

\begin{tabular}{|c|c|c|c|c|c|}
\hline \multirow{2}{*}{$t$} & \multicolumn{5}{|c|}{$\left\|e^{n}\right\|_{\infty, h}$} \\
\hline & Compact Scheme & Shao et al. [7] & C-N scheme & Raslan [10] & Dogan [11] \\
\hline 0.2 & $1.2536 \times 10^{-5}$ & 0.00056 & 0.00070 & 0.00190 & 0.00053 \\
\hline 0.4 & $2.4916 \times 10^{-5}$ & 0.00085 & 0.03331 & 0.00283 & 0.00113 \\
\hline 0.6 & $3.7265 \times 10^{-5}$ & 0.00112 & 0.06337 & 0.00403 & 0.00175 \\
\hline 0.8 & $4.9526 \times 10^{-5}$ & 0.00141 & 0.08433 & 0.00481 & 0.00237 \\
\hline 1.0 & $6.1667 \times 10^{-5}$ & 0.00169 & 0.11287 & 0.00563 & 0.00299 \\
\hline
\end{tabular}

TABLE 5: Comparison of $\|e\|_{\infty, h}$ by the compact scheme for $\sigma=1.03$, $\theta=0.25, p=2, \tau=h=0.1$.

\begin{tabular}{lccc}
\hline & & $\left\|e^{n}\right\|_{\infty, h}$ \\
$t$ & $\begin{array}{c}\text { Compact } \\
\text { Scheme }\end{array}$ & $\begin{array}{c}\text { Bakodah and Banaja } \\
{[6]}\end{array}$ & $\begin{array}{c}\text { Kutluay and Esen } \\
{[8]}\end{array}$ \\
\hline 4 & $7.0938 \times 10^{-5}$ & $1.4805 \times 10^{-4}$ & $1.23 \times 10^{-4}$ \\
8 & $2.1660 \times 10^{-4}$ & $2.9961 \times 10^{-4}$ & $1.66 \times 10^{-4}$ \\
12 & $5.1012 \times 10^{-4}$ & $4.5367 \times 10^{-4}$ & $1.79 \times 10^{-4}$ \\
\hline
\end{tabular}

that is,

$$
\begin{gathered}
\frac{1}{2}\left(\left\|e^{n+1}\right\|_{h}^{2}-\left\|e^{n-1}\right\|_{h}^{2}\right)+\frac{\beta}{2}\left(\left\|\delta_{x}^{+} e^{n+1}\right\|_{h}^{2}-\left\|\delta_{x}^{+} e_{x}^{n-1}\right\|_{h}^{2}\right) \\
\leq \tau K_{5}\left(\left\|e^{n+1}\right\|_{h}^{2}+\left\|e^{n}\right\|_{h}^{2}+\left\|e^{n-1}\right\|_{h}^{2}+\left\|\delta_{x}^{+} e^{n+1}\right\|_{h}^{2}\right. \\
\left.+\left\|\delta_{x}^{+} e^{n}\right\|_{h}^{2}+\left\|\delta_{x}^{+} e^{n-1}\right\|_{h}^{2}\right)+\tau\left\|r^{n}\right\|_{h}^{2},
\end{gathered}
$$

where $K_{5}=\max \left\{(1+\theta) / 2+K_{4},(9 / 2) K_{4}, 3 \theta\right\}$. Let

$$
B^{n}=\frac{1}{2}\left(\left\|e^{n+1}\right\|_{h}^{2}+\left\|e^{n}\right\|_{h}^{2}\right)+\frac{\beta}{2}\left(\left\|\delta_{x}^{+} e^{n+1}\right\|_{h}^{2}+\left\|\delta_{x}^{+} e^{n}\right\|_{h}^{2}\right) ;
$$

then (53) can be rewritten as

$$
B^{n}-B^{n-1} \leq \tau\left\|r^{n}\right\|_{h}^{2}+\tau K_{6}\left(B^{n}+B^{n-1}\right),
$$

where $K_{6}=\max \left\{2 K_{5}, 2 K_{5} / \beta\right\}$. From Lemma 8 , we have

$$
B^{N} \leq\left(B^{0}+T \sup _{1 \leq n \leq N}\left\|r^{n}\right\|_{h}^{2}\right) e^{4 K_{6} T} .
$$

Thus we can choose a fourth-order method to compute $u^{1}$ such that

$$
B^{0} \leq\left[O\left(\tau^{2}+h^{4}\right)\right]^{2}
$$

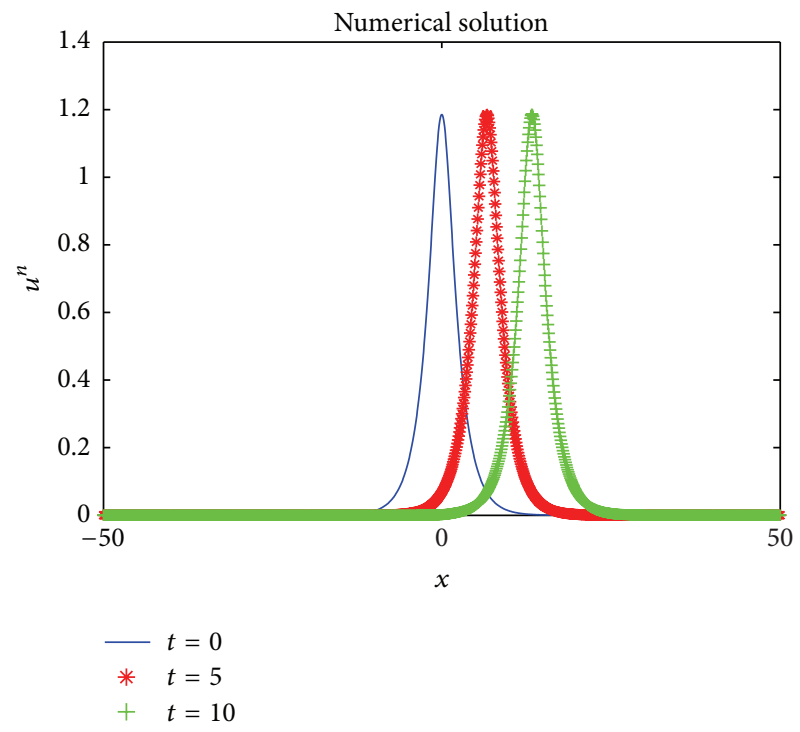

FIgURE 4: Numerical solution $u^{n}$ of scheme with $p=4$ and $\tau=h=$ 0.1 .

it follows from (56) that

$$
\left\|e^{n}\right\|_{h} \leq O\left(\tau^{2}+h^{4}\right), \quad\left\|\delta_{x}^{+} e^{n}\right\|_{h} \leq O\left(\tau^{2}+h^{4}\right),
$$

and then, from Lemma 6 , we obtain

$$
\|e\|_{\infty, h} \leq O\left(\tau^{2}+h^{4}\right) \text {. }
$$

This completes the proof.

Below, we can similarly prove stability of the difference solution.

Theorem 14. Under the conditions of Theorem 13, the solution of conservative finite difference scheme (25) is stable by $L^{\infty}$ norm. 


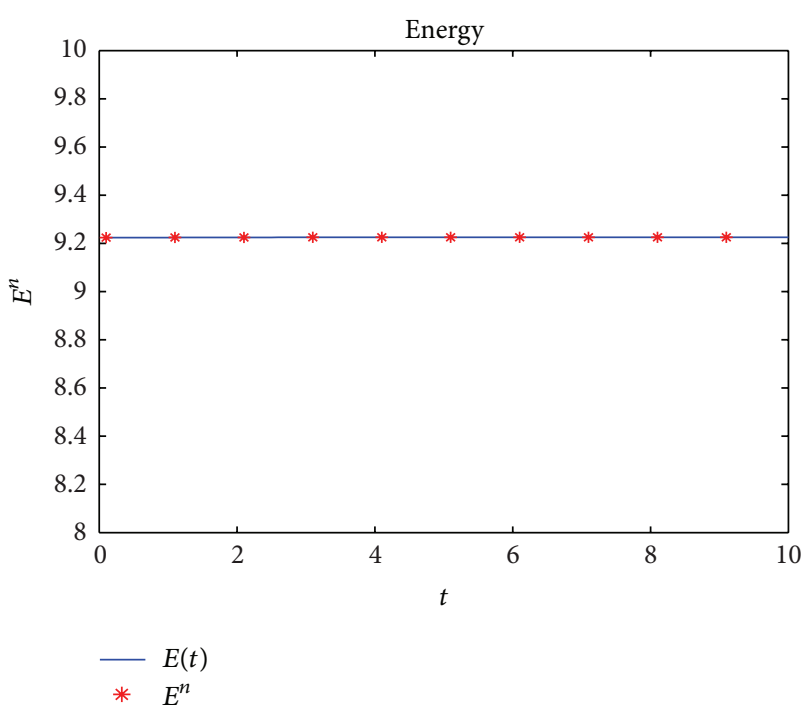

FIgURE 5: Discrete energy $E^{n}$ of scheme with $p=4, T=10$, and $\tau=h=0.1$.

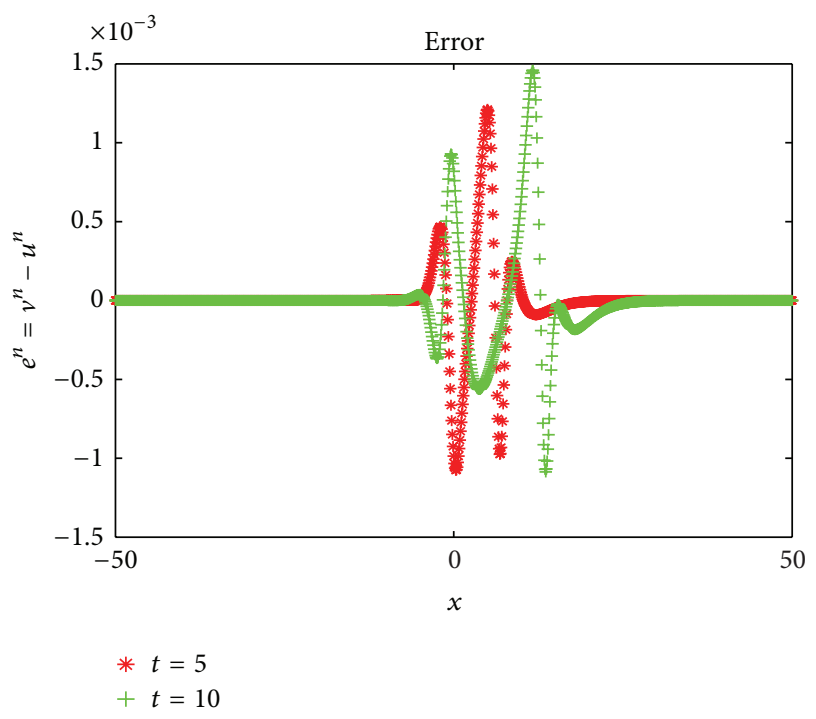

FIgURE 6: Absolute error $e^{N}$ of scheme with $p=4$ and $\tau=h=0.1$.

\section{Numerical Experiments}

In this section, two examples are presented to illustrate the effectiveness of the finite difference scheme $(25)$ in $[-50,50]$. The single solitary wave solution of (1) is

$$
u(x, t)=A \cdot \operatorname{sech}^{2 /(p-1)}\left[k\left(x+x_{0}-\sigma t\right)\right],
$$

where

$$
A=\left[\frac{(p+1)(\sigma-1)}{2 \alpha}\right]^{1 /(p-1)}, \quad k=\frac{p-1}{2 \beta} \sqrt{\frac{\sigma-1}{\sigma}},
$$

and $\sigma, x_{0}$ are arbitrary constants and $p \geq 2$.

Let $x_{0}=0$ in (60), $\alpha=1 / 2, \beta=1$, and $u_{0}(x)=A$. $\operatorname{sech}^{2 /(p-1)}(k x)$ and consider two cases: $p=2$ and $p=4$. We take $h=0.1$ and $\tau=0.1$, respectively. The errors $\left\|e^{n}\right\|_{\infty, h}$ are listed in Tables 1-2, respectively.

In Table 3,4 , and 5 , the comparison of $\|e\|_{\infty, h}$ by the compact scheme for $h=\tau=0.1$ with the Zhang [4] scheme for $h=0.05, \tau=0.1$ when $\sigma=4 / 3, \theta=0.25$ is shown. From Table 3, we can see that our compact scheme is acceptable.

Numerical results show that numerical precision depends on the choice of parameter $\theta$. From Tables 1-2, $\left\|e^{n}\right\|_{\infty, h} \leq$ $O\left(\tau^{2}+h^{4}\right)$ is validated. We take different $p, h$, and $\theta$ values and compute the errors for the solution of problem (1)(3). Numerical results are almost identical with the above experiment result. Hence, our schemes are efficient and reliable.

In Figures 1, 2, 3, 4, 5, and 6, we show the numerical solution and conservative discrete energy in each case.

\section{Conflict of Interests}

The authors declare that there is no conflict of interests regarding the publication of this paper.

\section{Acknowledgments}

This research was supported by the National Natural Science Foundation of China (Grant no. 11401183) and Fundamental Research Funds for the Central Universities.

\section{References}

[1] D. H. Peregrine, "Calculations of the development of an unduiar bore," Journal of Fluid Mechanics, vol. 25, pp. 321-330, 1966.

[2] D. H. Peregrine, "Long waves on a beach," Journal of Fluid Mechanics, vol. 27, no. 4, pp. 815-827, 1967.

[3] J. L. Bona, W. G. Pritchard, and L. R. Scott, "Numerical schemes for a model for nonlinear dispersive waves," Journal of Computational Physics, vol. 60, no. 2, pp. 167-186, 1985.

[4] L. Zhang, "A finite difference scheme for generalized regularized long-wave equation," Applied Mathematics and Computation, vol. 168, no. 2, pp. 962-972, 2005.

[5] Z. Ren, W. Wang, and D. Yu, "A new conservative finite difference method for the nonlinear regularized long wave equation," Applied Mathematical Sciences, vol. 5, no. 41-44, pp. 2091-2096, 2011.

[6] H. O. Bakodah and M. A. Banaja, "The method of lines solution of the regularized long-wave equation using Runge-Kutta time discretization method," Mathematical Problems in Engineering, vol. 2013, Article ID 804317, 8 pages, 2013.

[7] X. Shao, G. Xue, and C. Li, "A conservative weighted finite difference scheme for regularized long wave equation," Applied Mathematics and Computation, vol. 219, no. 17, pp. 9202-9209, 2013.

[8] S. Kutluay and A. Esen, "A finite difference solution of the regularized long-wave equation," Mathematical Problems in Engineering, vol. 2006, Article ID 85743, 14 pages, 2006.

[9] A. Esen and S. Kutluay, "Application of a lumped Galerkin method to the regularized long wave equation," Applied Mathematics and Computation, vol. 174, no. 2, pp. 833-845, 2006.

[10] K. R. Raslan, "A computational method for the regularized long wave (RLW) equation," Applied Mathematics and Computation, vol. 167, no. 2, pp. 1101-1118, 2005. 
[11] A. Dogan, "Numerical solution of RLW equation using linear finite elements within Galerkin's method," Applied Mathematical Modelling, vol. 26, no. 7, pp. 771-783, 2002.

[12] T. Roshan, "A Petrov-Galerkin method for solving the generalized regularized long wave (GRLW) equation," Computers \& Mathematics with Applications, vol. 63, no. 5, pp. 943-956, 2012.

[13] R. Mokhtari and M. Mohammadi, "Numerical solution of GRLW equation using sinc-collocation method," Computer Physics Communications, vol. 181, no. 7, pp. 1266-1274, 2010.

[14] C. M. Garcia-Lopez and J. I. Ramos, "Effects of convection on a modified GRLW equation," Applied Mathematics and Computation, vol. 219, no. 8, pp. 4118-4132, 2012.

[15] X. Li, L. Zhang, and S. Wang, "A compact finite difference scheme for the nonlinear Schrödinger equation with wave operator," Applied Mathematics and Computation, vol. 219, no. 6, pp. 3187-3197, 2012.

[16] T. Wang, B. Guo, and Q. Xu, "Fourth-order compact and energy conservative difference schemes for the nonlinear Schrödinger equation in two dimensions," Journal of Computational Physics, vol. 243, pp. 382-399, 2013.

[17] T. Wang and B. Guo, "Unconditional convergence of two conservative compact difference schemes for nonlinear Schroinger equation in one dimension," Scientia Sinica Mathematica, vol. 41, no. 3, pp. 207-233, 2011 (Chinese).

[18] Y. Zhou, Applications of Discrete Functional Analysis to the Finite Difference Method, International Academic, Beijing, China, 1990.

[19] T. Wang, L. Zhang, and F. Chen, "Conservative schemes for the symmetric regularized long wave equations," Applied Mathematics and Computation, vol. 190, no. 2, pp. 1063-1080, 2007. 


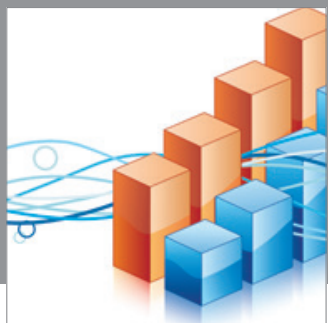

Advances in

Operations Research

mansans

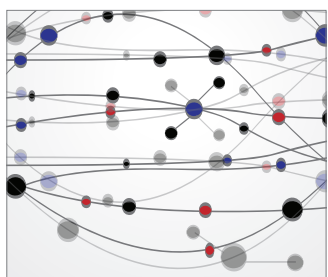

The Scientific World Journal
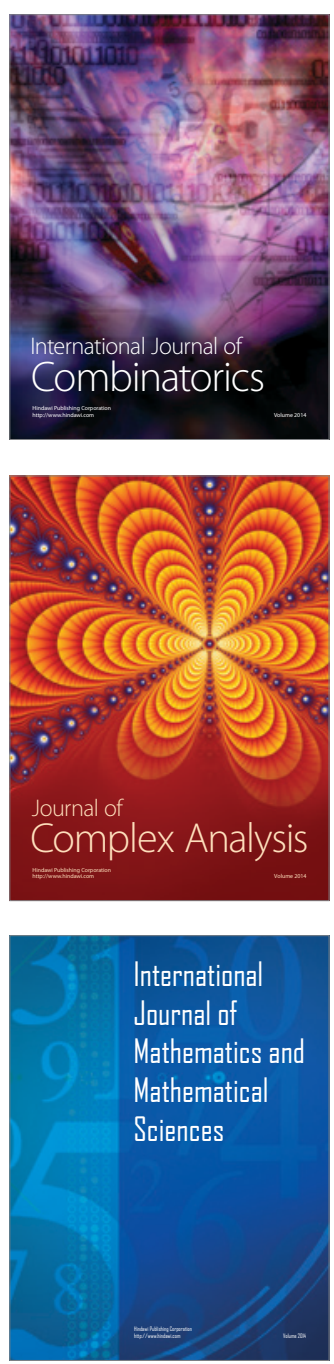
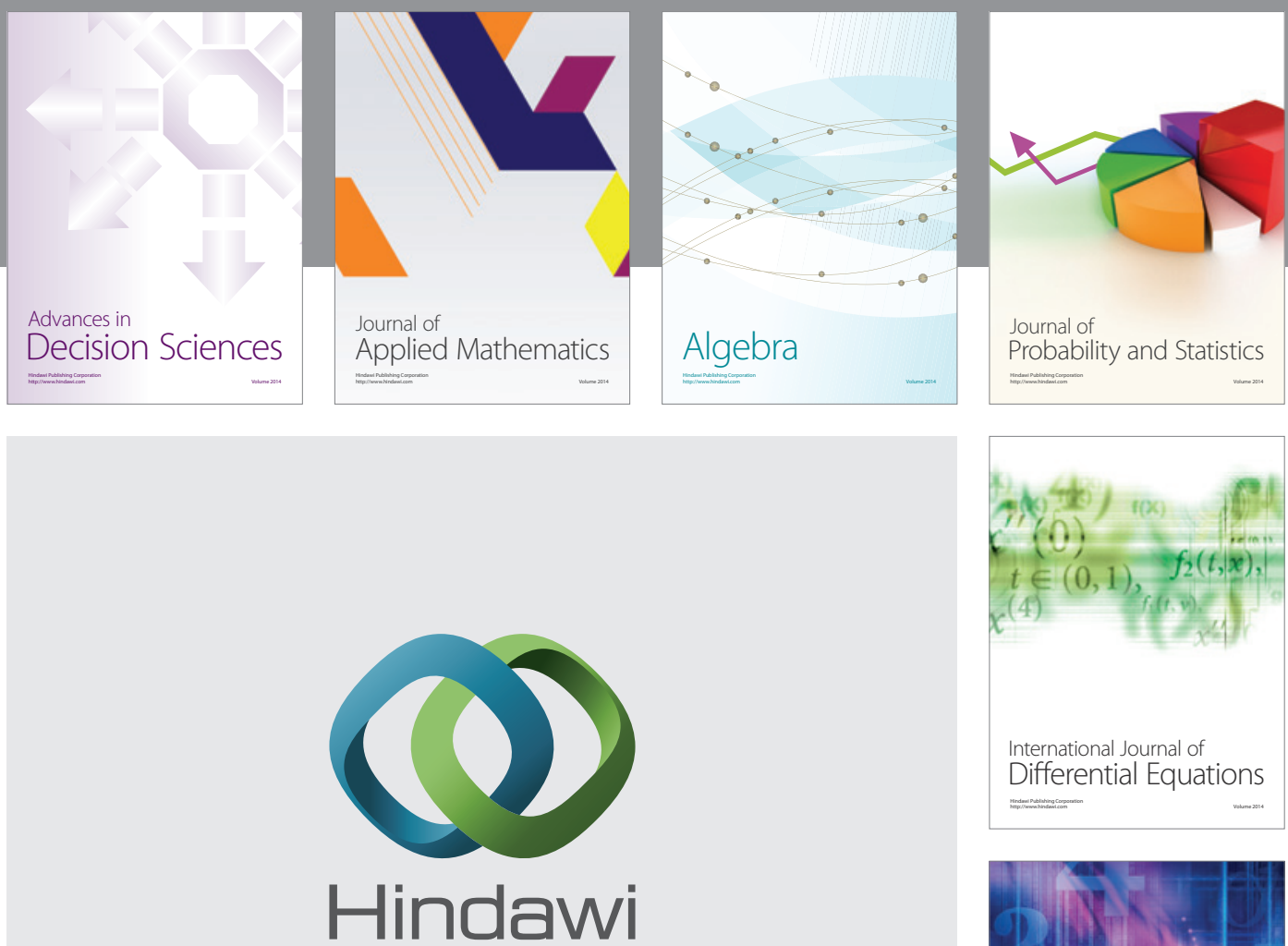

Submit your manuscripts at http://www.hindawi.com
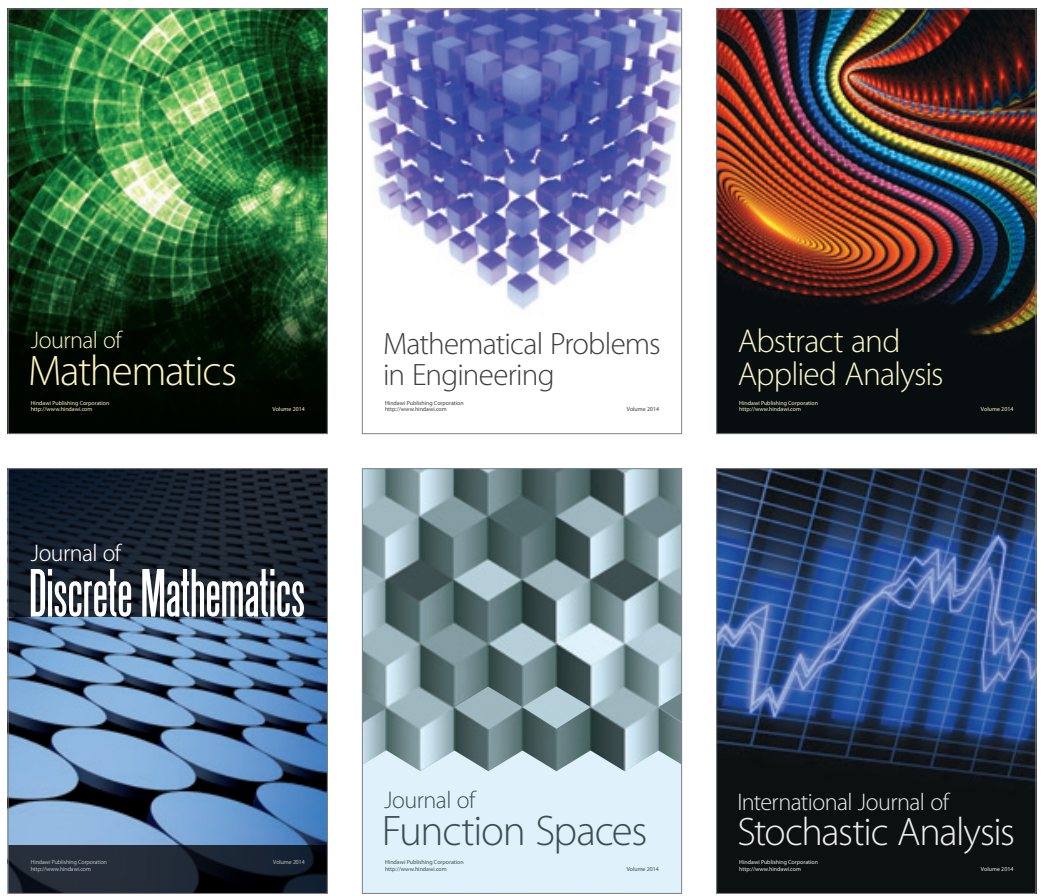

Journal of

Function Spaces

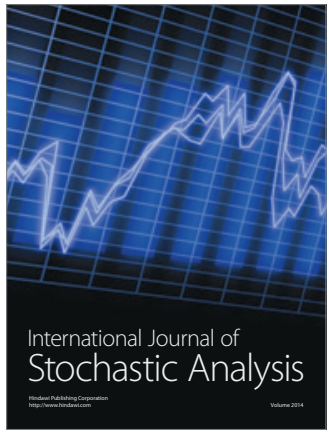

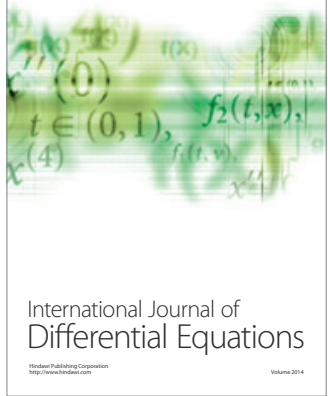
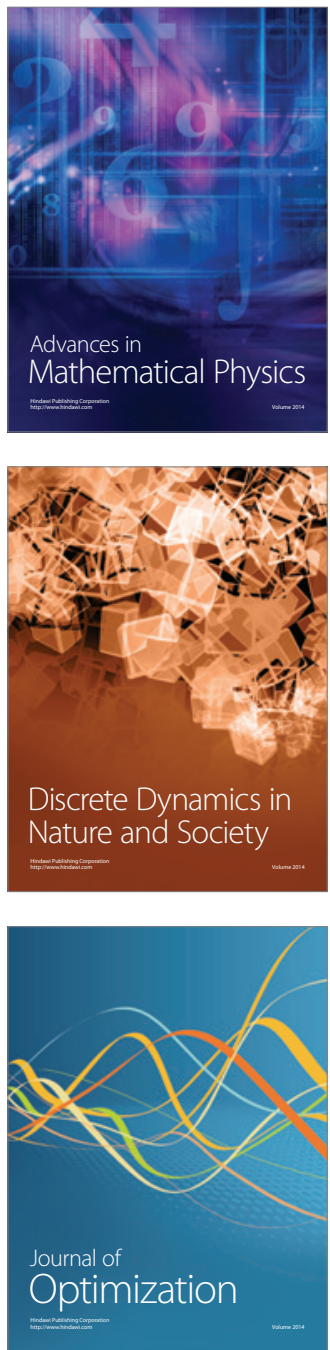